\title{
PHYSICA
}

Physica A 246 (1997) 199-220

\section{The role of the Manhattan distance in antiferromagnetic ordering}

\author{
G.J. Tielen ${ }^{a}$, T. Lulek ${ }^{b}$, M.R.M.J. Traa ${ }^{c}$, M. Kuzma ${ }^{c}$, W.J. Caspers ${ }^{\text {d,* }}$ \\ a Zelhem, The Netherlands \\ ${ }^{b}$ Institute of Physics, Adam Mickiewicz University, Poznan, Poland \\ c Institute of Physics, Pedagogical University, Rzeszow, Poland \\ d Center for Theoretical Physics, University of Twente, P.O. Box 217, 7500 AE Enschede, \\ The Netherlands
}

Received 8 April 1997

\begin{abstract}
The lowest state of one- and two-dimensional antiferromagnetic Heisenberg systems for a given number of "up" and "down" spins shows preference for certain regular patterns of the minority spin direction. For the one-dimensional system this pattern is an even distribution with equal distances for the minority spins, whereas in the two-dimensional systems the Manhattan distance, instead of the Euclidean distance, determines the optimal configuration.
\end{abstract}

PACS: 75.10.-b; 75.10.JM; 75.40.Mg

Keywords: Antiferromagnetic order; Manhattan distance

\section{Introduction}

The Bethe-Hulthén solution [1,2] for the ground state of the antiferromagnetic Heisenberg chain gives an algebraic representation of a state with a relatively low energy, from which the physical properties are not immediately evident. From an independent source, the work of Mermin and Wagner [3], it is known that this "ordered" state does not show a long-range order, as a consequence of global geometrical properties of a one-dimensional system, in particular, the shape of the $k$-spectrum of fluctuations, i.e., those with long wavelength destroy the long-range order, also for temperature zero.

In the first place this paper is about the remnants of order that exist in the Bethe-Hulthén solution for the ground state and which can be visualized in an easy

\footnotetext{
* Corresponding author. Tel.:0031 534893153/4893157; fax: 0031534891101 ; e-mail: w.j.caspers@thn.tn. utwente.nl.
} 
way: This solution shows a preference for an "even" distribution of both spin directions, "up" and "down" for a given quantization axis. (Both directions have the same number of representatives in all the Ising configurations contributing to the ground state.) This property may be readily generalized for the states of lowest energy for a given resulting $z$-component of the total spin, or a given number of "up" and "down" spins. In this case there is e.g. a minority of spins in the "down" direction and this minority also tries to realize an "even" distribution, i.e. a distribution with equal distances between successive spins in the down direction. The minority spins behave like a one-dimensional gas with a repulsive force between neighbour molecules, corresponding with the secular part of the energy. All configurations of the "molecules" are present in the ground state but the even distribution is the favoured one. The distribution with exactly equal distances cannot be realized in most cases as a consequence of the discrete character of the lattice, but the system tries to adhere to the rule as close as possible.

In the (quasi)classical Heisenberg model only the secular part of the energy plays a role and the energy is determined by the number of antiparallel neighbour pairs.

In the quantummechanical model also the nonsecular part has to be taken into consideration because it realizes the possibility of the exchange of a + and a - spin on neighbour positions, resulting in the occurrence of all configurations in the ground state, with the said preference for the even distribution.

Section 2 of this paper is devoted to the algebraic derivation of these properties of the one-dimensional Heisenberg system. In Section 3 we look for a generalization of these properties for two-dimensional lattices by determining the lowest stationary state for a given number of up and down spins on the square and the triangular lattice. For the square lattice we took squares of $4 \times 4$ and $6 \times 6$ lattice points with periodic boundary conditions, whereas for the triangular lattice calculations were done for a parallelogram of $4 \times 4$ points, also with periodic boundary conditions. In particular, we studied the case of 4 "down" spins in a background of $12(4 \times 4$ case $)$, respectively, $32(6 \times 6$ case) "up" spins. The numerical results were determined with the Lanczos method.

For the square lattice it turned out that the system shows a preference for configurations in which the 4 minority spins are ordered in a parallelogram instead of the more symmetrical square. This "even" distribution, the square, can be realized on the lattice in the two cases: $4 \times 4$ and $6 \times 6$, and it is the one which one would expect as the preferred configuration, on simple grounds of symmetry. A further inspection of the lowest energy states shows that the so-called Manhattan distance plays a crucial role in the amplitude of the different eigenstates of all the $z$-components of the individual spins, the so-called Ising states, or Ising configurations. The Manhattan distance between 2 lattice points on the square lattice is the length of the shortest path one has to follow on the lattice going step by step from one lattice point to a neighbouring one. The configurations with the largest amplitudes (in absolute value) represent states with a maximum sum of the 6 Manhattan distances between the 4 minority spins with the additional condition that the Manhattan distances between all possible pairs are evenly distributed, i.e. as close as possible in value. With this additional condition the relation between the absolute value of the amplitude and the Manhattan sum is 
a monotonous one. For the triangular lattice we could not determine a general rule that relates the Manhattan distances with the amplitude of the Ising configurations, apart from the maximum in absolute value corresponding with a pattern of equilateral triangles. This configuration corresponds with a maximum for the sum of generalized Manhattan distances on the triangular lattice.

The configuration in which the minority spins are ordered in a square on the square lattice represents an Ising state with maximum Manhattan sum, but the individual distances do not show the property of "evenness", they have larger differences than in the configurations with parallelograms.

So far we could not derive a general proof of the property that these special configurations in both type of lattices have the largest probability in the quantum ground state for a given total $z$-component of the spin. It is evident, however, that in the secular problem for the Heisenberg states, blocks of states with identical Manhattan sum play a crucial role: The Hamiltonian, in the case of small density of minority spins, demonstrates the selection rule that this sum can only change by \pm 1 , for an even number of these minority spins. This suggests the preference for the largest sum in the stationary state. It will also be clear that states with minority spins on neighbour positions are avoided because of their higher secular energy.

The states with an even distribution show the largest possible distance from these states with high secular energy, distance in terms of "Manhattan steps" of the minority spins, and for that reason these possibly have the largest amplitude in the ground state.

Finally, in Section 4 we discuss the isomorphism of the $4 \times 4$ system with the $2 \times 2 \times 2 \times 2$ system, i.e. a four-dimensional cube, in terms of which certain peculiarities of the ground state of the $4 \times 4$ system may be easily understood. Some groups of Ising amplitudes for this system turned out to be equal, a property that immediately follows from the symmetry of the four-dimensional hypercube.

\section{The "even" distribution of minority spins for the chain}

A very convenient presentation of the Bethe-Hulthén result is given by Thompson in Ref. [4]. In essence this result may be represented by a set of "deviations" from the ferromagnetic state, which deviations appear as running waves. These waves are characterized by wave numbers $k$, which may be interchanged between successive deviations along the chain. A stationary state is fully determined by its set of wave numbers and all distributions of this set over the deviations are included in this state. The wave numbers obey a set of algebraic equations that, for the general case, can only be solved numerically.

In this paper we restrict ourselves to $k$-spectra symmetrical with respect to $\pi$, which spectra represent a real state (apart from a trivial constant overall phase factor). Periodic boundary conditions imply that the antiferromagnetic chain is a ring and to avoid "frustration", which makes it impossible to realize an antiferromagnetic ordered state, we restrict ourselves to an even number of spins in the ring. This number will be $2 N$. 
By ordered state we always mean in this paper a state obeying Marshall's rule [11], which corresponds to a minimum in the energy for a given number of up and down spins.

The Hamiltonian of the antiferromagnetic chain:

$$
H_{\text {Heis }}=\sum_{n=1}^{2 N}\left(4 S_{n} \cdot S_{n+1}-1\right) \quad\left(S_{2 N+1} \equiv S_{1}\right)
$$

is chosen in such a way that the ferromagnetic state, represented by

$$
|+++\cdots+\rangle
$$

corresponds with energy $E=0$. This state is an eigenstate of all $S_{n}^{z}$ with eigenvalue $1 / 2(\hbar=1)$.

Stationary states with $r$ deviations ( $r$ reflecting plane waves) are represented by

$$
\Psi_{r}\left(k_{1}, k_{2}, k_{3}, \ldots k_{r}\right)=\sum_{1 \leqslant n_{1}<n_{2}<n_{3}<\cdots<n_{r} \leqslant 2 N}-a\left(n_{1}, n_{2}, n_{3}, \ldots n_{r}\right) \Phi_{n_{1} n_{2} n_{3} \ldots n_{r}},
$$

in which formula $\Phi_{n_{1} n_{2} n_{3} \ldots n_{r}}$ is a state with deviations at positions: $n_{1}, n_{2}, n_{3}, \ldots, n_{r}$ :

$$
\Phi_{n_{1} n_{2} n_{3} \ldots n_{r}}=S_{n_{1}}^{-} S_{n_{2}}^{-} S_{n_{3}}^{-} \ldots S_{n_{r}}^{-}|+++\cdots+\rangle\left(S_{n}^{-}=S_{n}^{x}-i S_{n}^{y}\right)
$$

and $a\left(n_{1}, n_{2}, n_{3}, \ldots n_{r}\right)$ is the corresponding amplitude:

$$
a\left(n_{1}, n_{2}, n_{3}, \ldots n_{r}\right)=\sum_{P} \exp i\left(\sum_{j=1}^{r} k_{P(j)} n_{j}+\frac{1}{2} \sum_{l<n} \phi_{P(l) P(n)}\right) .
$$

This amplitude is a superposition of products of plane waves for which the deviations at position $n_{j}$ all have their own wave number, which is one of a given set $\left(k_{1}, k_{2}, k_{3}, \ldots k_{r}\right)$. All permutations $P$, i.e. all distributions of the set of wave numbers over the deviations are included in the sum $\sum_{P}$. The interchange of a pair of wave numbers $\left(k_{j}, k_{l}\right)$ between two neighbour deviations is accompanied by a characteristic phase difference given by

$$
\cot \frac{\phi_{j l}}{2}=\frac{\sin \left(\left(k_{j}-k_{l}\right) / 2\right)}{\cos \left(\left(k_{j}+k_{l}\right) / 2\right)-\cos \left(\left(k_{j}-k_{l}\right) / 2\right)}, \quad \phi_{j l}=-\phi_{l j} .
$$

This phase difference is typical for the Bethe-Hulthén solution and Eq. (6) may be interpreted as a reflection condition that is a consequence of the fact that 2 deviations cannot occupy the same lattice site $[1,2,4]$.

The solution is completed by proper boundary conditions that determine the set of wave numbers $\left(k_{1}, k_{2}, k_{3}, \ldots k_{r}\right)$, which we take to be ordered in the following way:

$$
0 \leqslant k_{1}<k_{2}<k_{3}<\cdots<k_{r}<2 \pi,
$$

from which choice it follows that the phase differences [Eq. (6)] may be taken to obey:

$$
0<\phi_{j l}<\pi \quad(j<l) .
$$


The boundary conditions are essentially dictated by the identity:

$$
\Psi_{r}\left(n_{2}, n_{3}, \ldots n_{r}, 2 N+n_{1}\right)=\Psi_{r}\left(n_{1}, n_{2}, \ldots, n_{r-1}, n_{r}\right),
$$

and are given by

$$
2 N k_{j}=2 \pi\left(\lambda_{j}\right)+\sum_{l \neq j)} \phi_{j l} \quad\left(\lambda_{j} \text { is integer }\right) .
$$

A visualization of the solution determined by Eq. (6) and Eq. (10) may be given by the asymptotic case $r \ll 2 N$, under the extra condition that all $k$-values are chosen in the neighbourhood of $\pi$. The value $\pi$ represents the lowest possible energy for a state with one deviation. In general, a deviation of wave number $k$ contributes a lowering of the energy $-4(1-\cos k)$ with respect to the ferromagnetic state, resulting in the energy of a state with $r$ deviations given by

$$
E_{r}\left(k_{1}, k_{2}, k_{3}, \ldots k_{r}\right)=-4 \sum_{j=1}^{r}\left[1-\cos \left(k_{j}\right)\right] .
$$

The relative ground state in the case of $r$ deviations will be found by taking all $k$-values as close to $\pi$ as possible, but it follows from Eqs. (5) and (6) and the fact that the amplitudes of the Ising configurations can be chosen to be real, that all wave numbers should be different: Identical wave numbers result in an amplitude $a=0$ because $\phi_{j l} \rightarrow \pi$ for $k_{j} \rightarrow k_{l}(j<l)$. The only exception to this rule is the value 0 for two or more wave numbers, which are not interesting in our case in which only states in the neighbourhood of the ground state are considered.

We already made the restriction to $k$-spectra symmetric with respect to $\pi$. Asymptotic values of the wave numbers $k$ for $N \rightarrow \infty$, are conveniently represented by the differences from the value $\pi$ and the same is true for $\phi_{j l}(j<l)$. So we define

$$
k_{n}=\pi+\chi_{n}
$$

and

$$
\phi_{j l}=\pi-\partial \phi_{j l} \quad(j<l) .
$$

In terms of these reduced variables, the relation Eq. (6) between the angles $\phi_{j l}$ and the wave numbers $k_{j}$ and $k_{l}$ reads:

$$
\tan \left(\frac{\partial \phi_{j l}}{2}\right)=-\frac{\sin \left(\left(\chi_{j}-\chi_{l}\right) / 2\right)}{\cos \left(\left(\chi_{j}+\chi_{l}\right) / 2\right)+\cos \left(\left(\chi_{j}-\chi_{l}\right) / 2\right)} \quad(j<l) .
$$

In first order in terms of $1 / N$, we now find for the reduced wave numbers $\chi_{j}$ :

$$
\begin{aligned}
& \chi_{j} \rightarrow \frac{1}{2 N}\left[2 \pi\left(\Lambda_{j}\right)+\sum_{l(\neq j)} \phi_{j l}^{(0)}\right], \quad \lambda_{j}=N+\Lambda_{j} \quad\left(\Lambda_{j} \text { is integer }\right) \\
& \left(\phi_{j l}^{(0)}=\pi \text { for } j<l, \phi_{j l}^{(0)}=-\pi \text { for } j>l\right)
\end{aligned}
$$


and

$$
\partial \phi_{j l} \rightarrow-\frac{\chi_{j}-\chi_{l}}{2} \quad(j<l)
$$

The symmetry of the $k$-spectrum with respect to $\pi$ is reflected in the symmetry of the $\chi$-spectrum with respect to 0 , and the same is true for the integers $\Lambda_{j}$. Careful inspection shows that the $\Lambda_{j}$ are ordered in the same way as the $\chi_{j}$ and that the difference between two successive $\Lambda_{j}$ obeys:

$$
\Lambda_{j+1}-\Lambda_{j} \geqslant 2
$$

We now suppose that the parameters $\lambda_{j}$ (or $\lambda_{j}$ ) are adiabatic constants, i.e. their value for the lowest level and for all excited states in a given order are independent of $N$. So if one has determined these parameters for asymptotic values of $N$, these are already fixed in the nonasymptotic regime. This is essentially the noncrossing rule for energy levels as a function of a parameter, which usually only applies to a Hamiltonian that is a linear function of the parameter [5-10]. The rule cannot be strictly proven in general, but in the underlying case we tested it in a number of simple examples. This noncrossing rule is illustrated by Figs. 1 and 2 .

From Eqs. (11), (14) and (15) it follows that the lowest eigenvalue of the energy in the asymptotic regime, for successive values of $r$ is given by

$$
\begin{aligned}
& r=1: \Lambda_{1}=0, \quad \chi_{1}=0 \quad(\text { exact result for all } N), \\
& r=2: \Lambda_{1}=-\Lambda_{2}=-1, \quad \chi_{1}=-\chi_{2} \rightarrow-\frac{\pi}{2 N}, \\
& r=3: \Lambda_{1}=-\Lambda_{3}=-2, \quad \Lambda_{2}=0, \quad \chi_{1}=-\chi_{3} \rightarrow-\frac{\pi}{N}, \quad \chi_{2}=0 .
\end{aligned}
$$

For general $r$, one finds

$$
\Lambda_{j}=-r-1+2 j, \quad \chi_{j} \rightarrow-\frac{\pi}{2 N}(r+1-2 j) .
$$

Especially this last formula for $\Lambda_{j}$ giving the symmetrical, "even" distribution of $\Lambda$ 's around the value 0 , is central in the proof of the preference of the linear antiferromagnetic Heisenberg chain for an "even" distribution of the coordinates $n_{j}(j=1, \ldots r)$. This preference is expressed as a stationary point of the reduced amplitude $c\left(n_{1}, n_{2}, n_{3} \ldots n_{r}\right)$ defined by

$$
a\left(n_{1}, n_{2}, n_{3} \ldots n_{r}\right)=\exp i \pi\left(n_{1}+n_{2}+n_{3} \ldots+n_{r}\right) \times c\left(n_{1}, n_{2}, n_{3} \ldots n_{r}\right) .
$$

Also for states different from the lowest energy state that have a symmetrical distribution of $A$ - and $\chi$-values the theorem of the stationary point of the amplitude is true.

In general, such a stationary point needs not correspond with an extremum of the reduced amplitude. The relative ground state for given $r$ is the state with integers $A$ as close to 0 as possible, which correspond with minimal variation in the reduced 

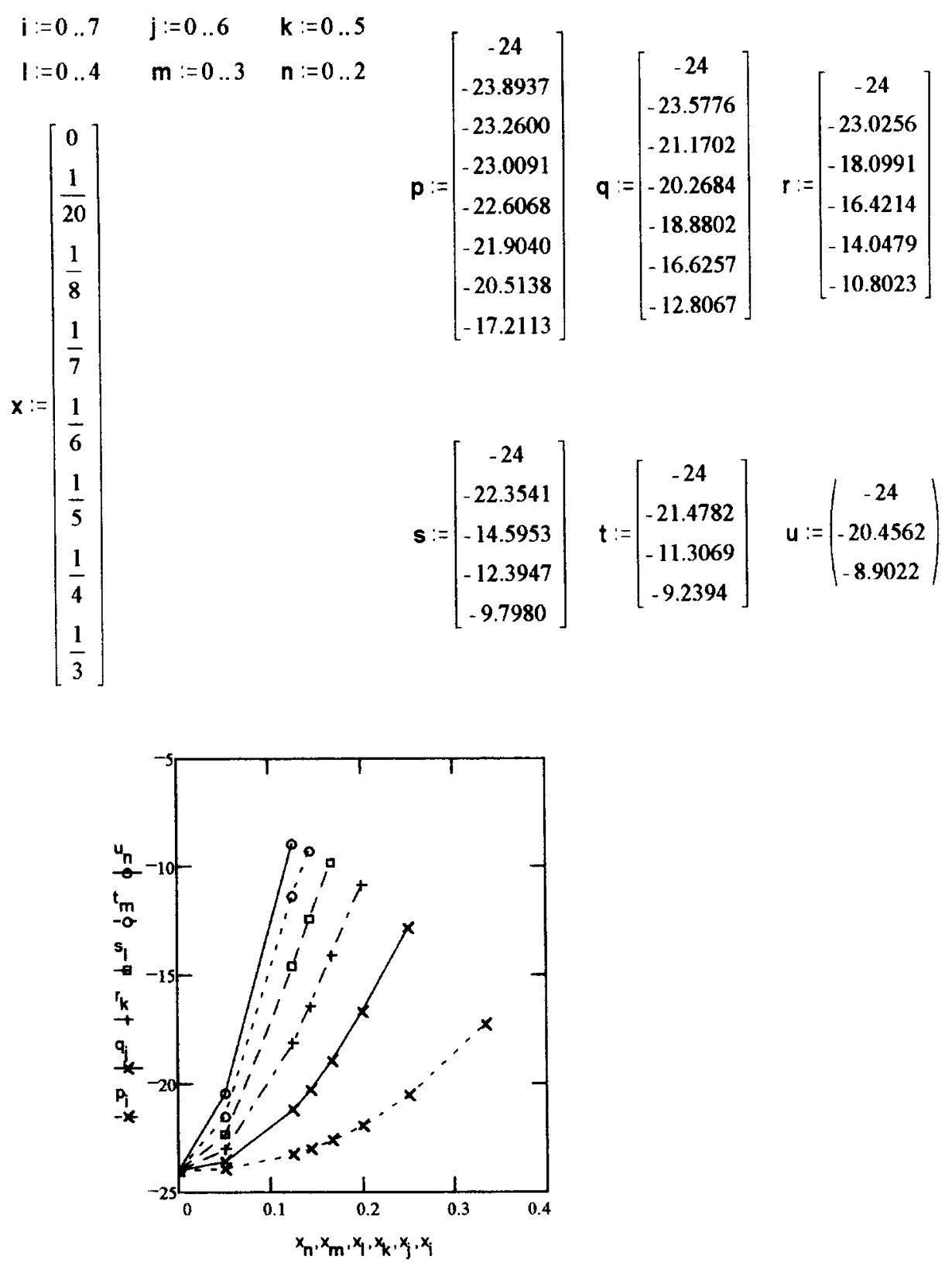

$p(\Lambda=-2) \quad q(\Lambda=-3) \quad r(\Lambda=-4) \quad s(\Lambda=-5) \quad t(\Lambda=-6) \quad u(\Lambda=-7)$

Fig. 1. Noncrossing levels for 3 deviations. 
$\mathrm{x}_{1}:=\left[\begin{array}{l}0 \\ \frac{1}{7} \\ \frac{1}{6} \\ \frac{1}{5} \\ \frac{1}{4}\end{array}\right] \quad \mathrm{x}_{2}:=\left[\begin{array}{l}0 \\ \frac{1}{7} \\ \frac{1}{6} \\ \frac{1}{5} \\ \frac{1}{4}\end{array}\right] \quad \mathrm{x}_{3}:=\left[\begin{array}{l}0 \\ \frac{1}{6} \\ \frac{1}{5} \\ \frac{1}{4}\end{array}\right] \quad \mathrm{p}:=\left[\begin{array}{c}-32 \\ -29.3723 \\ -28.2825 \\ -26.3691 \\ -22.6041\end{array}\right] \mathrm{q}:=\left[\begin{array}{c}-32 \\ -23.6881 \\ -20.6151 \\ -15.8084\end{array}\right] \mathrm{r}:=\left[\begin{array}{c}-32 \\ -25.7029 \\ -23.3886 \\ -19.7550\end{array}\right]$

$\mathbf{i}:=0 . .4 \quad \mathrm{j}:=0 . .3 \quad k:=0 . .2$

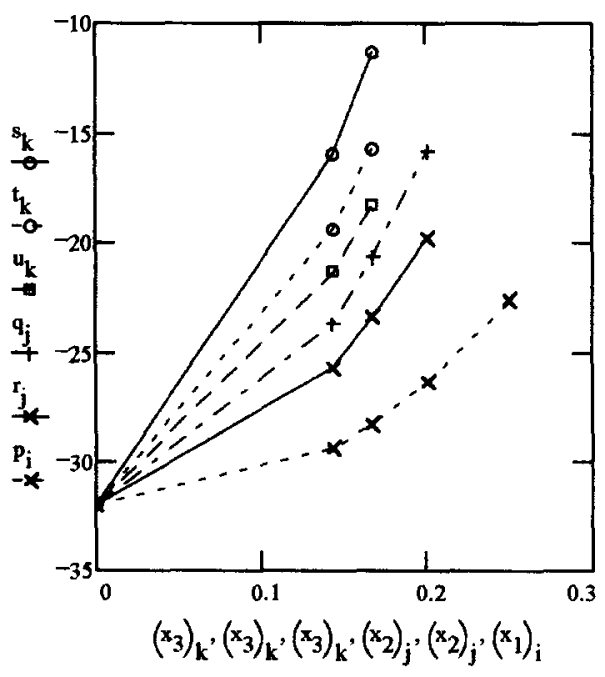

$$
\begin{array}{lll}
p=\left(\Lambda_{1}=-3, \Lambda_{2}=-1\right) & q=\left(\Lambda_{1}=-4, \Lambda_{2}=-2\right) & r=\left(\Lambda_{1}=-4, \Lambda_{2}=-1\right) \\
s=\left(\Lambda_{1}=-5, \Lambda_{2}=-3\right) & t=\left(\Lambda_{1}=-5, \Lambda_{2}=-2\right) & u=\left(\Lambda_{1}=-5, \Lambda_{2}=-1\right)
\end{array}
$$

Fig. 2. Noncrossing levels for 4 deviations.

amplitude. For this state the stationary point represent an extremum, and it is the only one apart from boundary extrema, given by, e.g.

$$
n_{j+1}=n_{j}+1 \text {, }
$$

for one or more of the set of consecutive coordinates:

$$
\left(n_{j}, n_{j+1}\right), \quad j=1,2,3 \ldots r, \quad n_{r+1} \equiv n_{1} .
$$


Theorem. The reduced amplitude $c\left(n_{1}, n_{2}, n_{3} \ldots n_{r}\right)$ of formula (18) has a stationary point for:

$$
n_{j}=n_{1,0}+(j-1) \frac{2 N}{r}, \quad j=1,2 \ldots r, \quad 1 \leqslant n_{1,0} \leqslant \frac{2 N}{r} .
$$

N.B.1: The coordinates of the stationary point are not necessarily integers, and consequently this point need not be realized for a realistic physical configuration.

N.B.2: Uniform translations of all $n_{j}$ result in a series of stationary points.

Proof. The proof may be given independently for all cyclic permutations of a given sequence of indices, $P(j), j=1,2, \ldots r$ i.e. for the corresponding partial sum in the reduced amplitude, given by Eqs. (5) and (18).

If we prove that all exponents within such a partial sum have the same value $(\bmod 2 \pi i)$ in the stationary point, the proof is completed, because differentiation with respect to one of the $n_{j}$ results in multiplication of the terms in the partial sum with one of the $\chi_{j}$. All terms correspond with a different $\chi_{j}$, all $\chi_{j}$ are included in the partial sum and they add up to 0 .

The general form of the exponents for two consecutive terms within the same set of cyclic permutations, at a supposedly stationary point $\left(n_{1,0}\right.$ is an arbitrary number in the interval $[0,2 N / r])$ :

$$
\left(n_{1,0}, n_{1,0}+\frac{2 N}{r}, n_{1,0}+2 \cdot \frac{2 N}{r}, \ldots n_{1,0}+(r-1) \frac{2 N}{r}\right),
$$

is

$$
\begin{gathered}
i\left\{\chi_{P(1)} n_{1,0}+\chi_{P(2)}\left[n_{1,0}+\frac{2 N}{r}\right]+\chi_{P(3)}\left[n_{1,0}+2 \cdot \frac{2 N}{r}\right]\right. \\
\left.+\ldots \chi_{P(r)}\left[n_{1,0}+(r-1) \frac{2 N}{r}\right]+\frac{1}{2} \sum_{n<l} \phi_{P(n) P(l)}\right\}
\end{gathered}
$$

and

$$
\begin{aligned}
& i\left\{\chi_{P(2)} n_{1,0}+\chi_{P(3)}\left[n_{1,0}+\frac{2 N}{r}\right]+\chi_{P(4)}\left[n_{1,0}+2 \cdot \frac{2 N}{r}\right]\right. \\
& \left.\quad+\ldots \chi_{P(1)}\left[n_{1,0}+(r-1) \frac{2 N}{r}\right]+\frac{1}{2} \sum_{n<1} \phi_{P(n+1) P(l+1)}\right\} .
\end{aligned}
$$

Because of the fact that $\left(\sum_{j} \chi_{P(j)}\right) 2 N / r=0$ the difference between these exponents equals:

$$
i\left[-2 N \chi_{P(1)}+\frac{1}{2} \sum_{n<1}\left(\phi_{P(n) P(l)}-\phi_{P(n+1) P(l+1)}\right)\right] .
$$

Taking into account the property of the cyclic permutations:

$$
P(r+1)=P(1),
$$


the expression for the difference may be rewritten:

$$
i\left(-2 N \chi_{P(1)}+\sum_{l>1} \phi_{P(1) P(l)}\right),
$$

which equals:

$$
-2 i \pi\left(\Lambda_{P(1)}\right) \quad\left(\Lambda_{P(1)} \text { is integer }\right),
$$

according to Eq. (10).

So all the exponentials in the partial sum have the same value at the point [Eq. (21)], which may now be considered to be a stationary point because the derivatives with respect to the single $n_{j}$ 's add up to 0 .

For the ground state for given $r$ this stationary point corresponds to an extremum, as will be illustrated for simple examples.

The existence of a stationary point may in general be explained by a symmetry argument.

The same is true for boundary extrema in the ground state, which occur if one of the differences $n_{j+1}-n_{j}$ has the minimum value: 1. The remaining coordinates will take values that result in a symmetrical configuration of the corresponding deviations with respect to the boundaries fixed by $n_{j}$ and $n_{j+1}$. If for instance in the case $r=3$, with

$$
1 \leqslant n_{1}<n_{2}<n_{3} \leqslant 2 N,
$$

the first and the third deviation are on neighbouring positions, the extremum should correspond with:

$$
n_{1}=1, \quad n_{2}=N+\frac{1}{2}, \quad n_{3}=2 N .
$$

Again this configuration does not represent a real state of the spin lattice, but it may help to determine the global structure of the state vector.

\subsection{Examples}

The relative ground state for all fixed values of $2 N$ and $r$ obeys the "criterion" originally formulated by Marshall [11]. This criterion states that interchanging a + and a - spin on neighbouring positions results in a change of sign of the corresponding amplitude. All the amplitudes can always be chosen to be real in the ground state. This change of sign is an expression of the fact that the spectrum of $k$-values is symmetrical with respect to $\pi$ and the different wave numbers have values as close to $\pi$ as possible (cf. (Eq. (18))). They could not all condense to this value, because they should be different, with the exception of the value 0 .

These general characteristics of the amplitudes are clearly illustrated by the following simple examples: 
One deviation: $r=1$. There is no reflection condition in this case and the complete solution of the Bethe-Hulthén problem is given by Eqs. (5) and (10). The solution is exact and is identical with its asymptotic form Eq. (14). There are no (reduced) phase angles. The amplitude is given by the formulas:

$$
\begin{aligned}
& a\left(n_{1}\right)=\exp \left(i n_{1} \pi\right) \exp \left(i \chi_{1} n_{1}\right), \\
& 2 N \chi_{1}=2 \Lambda_{1} \pi, \quad \Lambda_{1}=0,1,2 . .2 N-1 .
\end{aligned}
$$

It will be immediately clear that the ground state is represented by

$$
\Lambda_{1}=0, \lambda_{1}=N \quad \text { and } \quad \chi_{1}=0, k_{1}=\pi,
$$

which state clearly obeys Marshall's criterion.

Two deviations: $r=2$. For the case of a symmetrical $k$-spectrum there are only 2 relevant parameters. The reduced wave numbers are given by the set:

$$
\left(\chi_{1}, \chi_{2}\right)=\left(\chi_{1},-\chi_{1}\right)
$$

and there is only one relevant phase angle:

$$
\phi_{12}=-\phi_{21}, \quad \phi_{12}=\pi-\partial \phi_{12} \text {. }
$$

The amplitude is given by

$$
a\left(n_{1}, n_{2}\right)=2 \exp \left[i\left(n_{1}+n_{2}\right) \pi\right] \cos \left[\chi_{1}\left(n_{1}-n_{2}\right)+\frac{1}{2} \phi_{12}\right] .
$$

The parameters are determined by Eqs. (6), (6a) and (10), or in the reduced form for $r=2$ :

$$
\tan \left(\frac{\partial \phi_{12}}{2}\right)=-\frac{\sin \left(\chi_{1}-\chi_{2}\right) / 2}{\cos \left(\chi_{1}+\chi_{2}\right) / 2+\cos \left(\chi_{1}-\chi_{2}\right) / 2}=-\frac{\sin \chi_{1}}{1+\cos \chi_{1}}=-\tan \left(\frac{\chi_{1}}{2}\right)
$$

and

$$
(2 N-1) \chi_{1}=2 \pi\left(\Lambda_{1}+\frac{1}{2}\right), \quad \Lambda_{1}=-N+1,-N+2, \ldots,-1 .
$$

From Eq. (34) and the general description of the Bethe-Hulthén solution it follows that

$$
0<\partial \phi_{12}=-\chi_{1}<\pi
$$

which relation is used in the derivation of Eq. (35) from formula Eq. (10).

According to Eq. (11) the lowest energy will be reached for $\Lambda_{1}=-1$ or:

$$
\chi_{1}=-\frac{\pi}{2 N-1}, \quad k_{1}=\pi-\frac{\pi}{2 N-1} .
$$

Now one easily shows that the second factor in Eq. (33) is negative for all values of $\left(n_{1}, n_{2}\right)$ in the range: $1 \leqslant n_{1}<n_{2} \leqslant 2 N$. Because of the fact that the first factor changes 
Table 1

Numerical solution for the Bethe-Hulthén problem for 4 deviations $(N=4,5)$.

\begin{tabular}{|c|c|c|c|c|c|c|c|c|}
\hline \multirow[t]{2}{*}{$\left(\Lambda_{1}, \Lambda_{2}\right)$} & \multicolumn{4}{|l|}{$N=4$} & \multicolumn{4}{|l|}{$N=5$} \\
\hline & $\partial \phi_{12}$ & $\partial \phi_{13}$ & $\chi_{1}$ & $\chi_{2}$ & $\partial \phi_{12}$ & $\partial \phi_{13}$ & $\chi_{1}$ & $\chi_{2}$ \\
\hline$(-3,-1)$ & 0.75331 & 1.15904 & -1.16959 & -0.50676 & 0.47219 & 0.82423 & -1.19124 & -0.38818 \\
\hline$(-4,-2)$ & no sol. & no sol. & no sol. & no sol. & 0.91265 & 1.69038 & -2.03456 & -1.13361 \\
\hline$(-4,-1)$ & no sol. & no sol. & no sol. & no sol. & 1.25981 & 1.48563 & -2.05038 & -0.37416 \\
\hline
\end{tabular}

Table 2

Numerical solution for the Bethe-Hulthén problem for 4 deviations $(N=6,7)$

\begin{tabular}{|c|c|c|c|c|c|c|c|c|}
\hline \multirow[t]{2}{*}{$\left(\Lambda_{1}, A_{2}\right)$} & \multicolumn{4}{|l|}{$N=6$} & \multicolumn{4}{|l|}{$N=7$} \\
\hline & $\partial \phi_{12}$ & $\partial \phi_{13}$ & $\chi_{1}$ & $\chi_{2}$ & $\partial \phi_{12}$ & $\partial \phi_{13}$ & $\chi_{1}$ & $\chi_{2}$ \\
\hline$(-3,-1)$ & 0.35156 & 0.64670 & -0.94755 & -0.31243 & 0.28247 & 0.53380 & -0.78777 & -0.26099 \\
\hline$(-4,-2)$ & 0.50986 & 1.30168 & -1.59268 & -0.92878 & 0.35999 & 1.07021 & -1.31832 & -0.77962 \\
\hline$(-4,-1)$ & 0.82455 & 1.06980 & -1.60021 & -0.30789 & 0.62618 & 0.85235 & -1.32204 & -0.25906 \\
\hline$(-5,-3)$ & 1.11176 & 2.00222 & -2.28228 & -1.50895 & 0.57351 & 1.61579 & -1.86003 & -1.28848 \\
\hline$(-5,-2)$ & 1.44044 & 1.87219 & -2.30034 & -0.89605 & 0.88902 & 1.44347 & -1.87105 & -0.76763 \\
\hline$(-5,-1)$ & 1.62069 & 1.75302 & -2.30590 & -0.29763 & 1.10574 & 1.28101 & -1.87522 & -0.25514 \\
\hline$(-6,-4)$ & no sol. & no sol. & no sol. & no sol. & 1.31832 & 2.20806 & -2.44621 & -1.76007 \\
\hline$(-6,-3)$ & no sol. & no sol. & no sol. & no sol. & 1.62615 & 2.11565 & -2.46278 & -1.24596 \\
\hline$(-6,-2)$ & no sol. & no sol. & no sol. & no sol. & 1.77961 & 2.03816 & -2.46862 & -0.74487 \\
\hline$(-6,-1)$ & no sol. & no sol. & no sol. & no sol. & 1.88195 & 1.96352 & -2.47075 & -0.24794 \\
\hline
\end{tabular}

sign for a shift $\Delta n_{1}= \pm 1$ or $\Delta n_{2}= \pm 1$ the (relative) ground state for 2 deviations is a state obeying Marshall's criterion.

Also for $r=3$ it is possible to prove exactly that the ground state as given by the Bethe-Hulthen analysis obeys Marshall's criterion, but the argument is rather longwinded. We prefer to give some numerical results for the case $r=4$ and to show the qualitatively different behaviour of the ground state and some excited states. Again we restrict ourselves to states with $\chi$-spectra symmetrical with respect to 0 , which include the ground state.

Four deviations: $r=4$. The spectrum of $\chi$-values now takes the form:

$$
\left(\chi_{1}, \chi_{2},-\chi_{2},-\chi_{1}\right) \text {, }
$$

and for reasons of symmetry there are only four independent reflection angles $\phi_{i j}$ :

$$
\left(\phi_{12}, \phi_{13}, \phi_{14}, \phi_{23}\right) \text {, }
$$

because $\phi_{12}=-\phi_{21}, \phi_{24}=\phi_{13}$, etc. It should also be clear that there are only two independent $A$-parameters:

$$
\left(A_{1} \Lambda_{2}\right)
$$


Table 3

Asymptotic solution for the Bethe-Hulthén problem for 4 deviations

\begin{tabular}{lllll}
\hline$\left(\Lambda_{1}, \Lambda_{2}\right)$ & $\partial \phi_{12}$ & $\partial \phi_{13}$ & $\chi_{1}$ & $\chi_{2}$ \\
\hline$(-3,-1)$ & $\pi / 2 N$ & $\pi / N$ & $-3 \pi / 2 N$ & $-\pi / 2 N$ \\
$(-4,-2)$ & $\pi / 2 N$ & $2 \pi / N$ & $-5 \pi / 2 N$ & $-3 \pi / 2 N$ \\
$(-4,-1)$ & $\pi / N$ & $3 \pi / 2 N$ & $-5 \pi / 2 N$ & $-\pi / 2 N$ \\
$(-5,-3)$ & $\pi / 2 N$ & $3 \pi / N$ & $-7 \pi / 2 N$ & $-5 \pi / 2 N$ \\
$(-5,-2)$ & $\pi / N$ & $5 \pi / 2 N$ & $-7 \pi / 2 N$ & $-3 \pi / 2 N$ \\
$(-5,-1)$ & $3 \pi / 2 N$ & $2 \pi / N$ & $-7 \pi / 2 N$ & $-\pi / 2 N$ \\
$(-6,-4)$ & $\pi / 2 N$ & $4 \pi / N$ & $-9 \pi / 2 N$ & $-7 \pi / 2 N$ \\
$(-6,-3)$ & $\pi / N$ & $7 \pi / 2 N$ & $-9 \pi / 2 N$ & $-5 \pi / 2 N$ \\
$(-6,-2)$ & $3 \pi / 2 N$ & $3 \pi / N$ & $-9 \pi / 2 N$ & $-3 \pi / 2 N$ \\
$(-6,-1)$ & $2 \pi / N$ & $5 \pi / 2 N$ & $-9 \pi / 2 N$ & $-\pi / 2 N$ \\
\hline
\end{tabular}

The unknown parameters to be determined:

$$
\partial \phi_{12}, \partial \phi_{13}, \partial \phi_{14}, \partial \phi_{23}, \chi_{1}, \chi_{2}, \Lambda_{1}, \Lambda_{2},
$$

are solutions of Eqs. (6) (6a) and (10), taking into account the definitions Eqs. (12) and (13). In Tables 1-6 we give numerical results for some low-lying states for $N=4,5,6,7$. The complete set of amplitudes is given for $N=4,5,6$, whereas for $N=7$ we only give the parameters $(41)$. The numerical calculations are simplified by the identities:

$$
\partial \phi_{14}=-\chi_{1}, \quad \partial \phi_{23}=-\chi_{2},
$$

which follow from Eq. (6a) and the symmetry of the $\chi$-spectrum, in the same way as the identity Eq. (36) for $r=2$. The number of stationary states depends on $N$ : That is why there are $A$-values in Tables 1-3 without a corresponding solution.

Because of translational symmetry i.e. the symmetry of the $\chi$-spectrum around the value 0 , the reduced amplitude only depends on the differences of the coordinates of the successive deviations, which will be denoted by $x, y$ and $z$ :

$$
x=n_{2}-n_{1}, \quad y=n_{3}-n_{2}, \quad z=n_{4}-n_{3} .
$$

\subsection{Discussion of results presented in Tables $1-6$}

Table 3 presents the asymptotic solution of the Bethe-Hulthén problem as given in Eqs. (14) and (15). The given values for the reduced phase angles and wave numbers may be compared with those of Tables 1 and 2 . In Table 4 the stationary point for the reduced amplitude as function of the coordinate differences is indicated by bold entries. Here the stationary point is an extremum because the results refer to the ground state. As stated before the extremum does not need to correspond to a realistic configuration because it may have non-integer coordinates. In an analogous way stationary points are marked in Tables 5 and 6 for the states represented by the parameter values:

$$
\left(\Lambda_{1}, \Lambda_{2}\right)=(-4,-1), \quad\left(\Lambda_{1}, \Lambda_{2}\right)=(-4,-2) \quad \text { and } \quad\left(\Lambda_{1}, \Lambda_{2}\right)=(-5,-3)
$$


Table 4

Reduced amplitudes for the Bethe-Hulthén solution: $c(x, y, z)$. Ground state

\begin{tabular}{|c|c|c|c|}
\hline \multirow[t]{2}{*}{$\left(\Lambda_{1}, \Lambda_{2}\right)=(-3,-1)$} & \multicolumn{3}{|l|}{$N$} \\
\hline & 4 & 5 & 6 \\
\hline$(x, y, z)=(1,1,1)$ & 0.14257 & 0.03134 & 0.00905 \\
\hline$(1,1,2)$ & 0.66312 & 0.17525 & 0.05492 \\
\hline$(1,1,3)$ & 1.04109 & 0.39764 & 0.14497 \\
\hline$(1,1,4)$ & & 0.50746 & 0.23748 \\
\hline$(1,1,5)$ & & & 0.27677 \\
\hline$(1,2,1)$ & 1.04109 & 0.30516 & 0.10012 \\
\hline$(1,2,2)$ & 3.13801 & 1.22610 & 0.45273 \\
\hline$(1,2,3)$ & 2.61746 & 1.93273 & 0.89499 \\
\hline$(1,2,4)$ & & 1.74328 & 1.16589 \\
\hline$(1,2,5)$ & & 0.87540 & 1.09155 \\
\hline$(1,2,6)$ & & & 0.72498 \\
\hline$(1,2,7)$ & & & 0.30282 \\
\hline$(1,3,1)$ & 1.71894 & 0.80951 & 0.32691 \\
\hline$(1,3,2)$ & & 2.49136 & 1.20488 \\
\hline$(1,3,3)$ & & 2.89757 & 1.95282 \\
\hline$(1,3,4)$ & & & 2.09195 \\
\hline$(1,3,5)$ & & & 1.55191 \\
\hline$(1,4,1)$ & & 1.08504 & 0.60176 \\
\hline$(1,4,2)$ & & & 1.84637 \\
\hline$(1,4,3)$ & & & 2.46333 \\
\hline$(1,5,1)$ & & & 0.72827 \\
\hline$(2,2,2)$ & 7.60225 & 4.48837 & 1.91588 \\
\hline$(2,2,3)$ & & 6.17404 & 3.50018 \\
\hline$(2,2,4)$ & & & 4.18389 \\
\hline$(2,3,2)$ & & 6.68573 & 4.11661 \\
\hline$(2,3,3)$ & & & 6.08876 \\
\hline$(2,3,4)$ & & & 5.78716 \\
\hline$(2,4,2)$ & & & 5.16857 \\
\hline$(3,3,3)$ & & & 7.93187 \\
\hline$(5 / 2,5 / 2,5 / 2)$ & & $\mathbf{7 . 8 5 8 1 2}$ & \\
\hline
\end{tabular}

respectively. The last set of parameters is only realized for $N \geqslant 6$. It should be clear that for the parameter set $\left(\Lambda_{1}, \Lambda_{2}\right)=(-4,-2)$ the stationary point is not an extremum.

With Eq. (11) one can calculate the energy eigenvalues for the states with a given parameter $\Lambda(r=3)$ or a parameter set $\left(\Lambda_{1}, \Lambda_{2}\right)(r=4)$ as a function of $x=1 / N$. It turns out that the curves for these eigenvalues do not intersect, as is illustrated by Figs. 1 and 2 . This is an example of the so-called non-crossing rule, in a slightly 
Table 5

Reduced amplitudes for the Bethe-Hulthén solution: $c(x, y, z)$. Excited states: $\left(\Lambda_{1}, \Lambda_{2}\right)=(-4,-1)$ and $\left(\Lambda_{1}, \Lambda_{2}\right)=(-4,-2)$

\begin{tabular}{|c|c|c|c|c|}
\hline \multirow[t]{2}{*}{$(x, y, z)$} & \multicolumn{2}{|c|}{$\left(\Lambda_{1}, \Lambda_{2}\right)=(-4,-1)$} & \multicolumn{2}{|c|}{$\left(\Lambda_{1}, A_{2}\right)=(-4,-2)$} \\
\hline & $N=5$ & $N=6$ & $N=5$ & $N=6$ \\
\hline$(1,1,1)$ & 0.28760 & 0.10054 & 0.18453 & 0.10153 \\
\hline$(1,1,2)$ & 1.13281 & 0.48735 & 0.54475 & 0.42173 \\
\hline$(1,1,3)$ & 1.36186 & 0.85920 & -0.07532 & 0.42755 \\
\hline$(1,1,4)$ & 1.03330 & 0.80044 & -0.87109 & -0.27525 \\
\hline$(1,1,5)$ & & 0.65608 & & -0.76521 \\
\hline$(1,2,1)$ & 1.41318 & 0.74924 & 1.41166 & 0.75115 \\
\hline$(1,2,2)$ & 3.02027 & 2.39505 & 2.21103 & 1.94729 \\
\hline$(1,2,3)$ & 1.67481 & 2.49464 & -1.62518 & 0.45088 \\
\hline$(1,2,4)$ & 2.81811 & 1.72360 & -2.17878 & -2.13810 \\
\hline$(1,2,5)$ & 3.59560 & 2.14884 & 0.60600 & -1.84945 \\
\hline$(1,2,6)$ & & 2.92807 & & 0.60311 \\
\hline$(1,2,7)$ & & 2.04082 & & 1.37991 \\
\hline$(1,3,1)$ & 0.85091 & 1.17024 & 2.95333 & 1.60402 \\
\hline$(1,3,2)$ & -0.51961 & 2.10703 & 3.55432 & 3.11155 \\
\hline$(1,3,3)$ & -0.19464 & 0.74741 & -2.36937 & 0.22722 \\
\hline$(1,3,4)$ & & 0.80942 & & -2.71216 \\
\hline$(1,3,5)$ & & 2.80578 & & -1.58572 \\
\hline$(1,4,1)$ & -0.35362 & 0.32901 & 3.64144 & 2.29487 \\
\hline$(1,4,2)$ & & -0.84129 & & 4.12613 \\
\hline$(1,4,3)$ & & -1.34368 & & 0.60900 \\
\hline$(1,5,1)$ & & -0.43736 & & 2.61657 \\
\hline$(2,2,2)$ & 2.81515 & 6.32391 & 0.94000 & 3.77483 \\
\hline$(2,2,3)$ & -2.67583 & 4.25092 & -3.63156 & -1.30634 \\
\hline$(2,2,4)$ & & 1.61883 & & -5.42433 \\
\hline$(2,3,2)$ & -6.80747 & 0.93275 & 3.22831 & 5.05183 \\
\hline$(2,3,3)$ & & -3.79375 & & -0.10226 \\
\hline$(2,3,4)$ & & -1.36816 & & -3.65994 \\
\hline$(2,4,2)$ & & -5.01864 & & 6.97466 \\
\hline$(3,3,3)$ & & -8.21546 & & -0.35451 \\
\hline$(5 / 2,5 / 2,5 / 2)$ & -8.45644 & & -0.91051 & \\
\hline
\end{tabular}

modified form. We do not claim that the customary arguments for non-crossing hold in this case and this point needs further study. But we may interpret this property again as an example of the rule that crossing only occurs as an exceptional phenomenon [5-10]. The non-crossing rule has the consequence in this case that the ground state corresponds with the parameter set $\left(\Lambda_{1}, \Lambda_{2}\right)=(-3,-1)$ for all $N$, as was already stated in the work of Bethe and Hulthén $[1,2]$. 
Table 6

Reduced amplitudes for the Bethe-Hulthén solution: $c(x, y, z)$. Excited states: $\left(\Lambda_{1}, \Lambda_{2}\right)=$ $(-5,-3)$

\begin{tabular}{lrrr}
\hline$(x, y, z)$ & $N=6$ & $(x, y, z)$ & $N=6$ \\
\hline$(1,1,1)$ & -0.03665 & $(1,4,1)$ & 4.14128 \\
$(1,1,2)$ & -0.06663 & $(1,4,2)$ & 2.05938 \\
$(1,1,3)$ & 0.16251 & $(1,4,3)$ & -5.32127 \\
$(1,1,4)$ & 0.06335 & & \\
$(1,1,5)$ & -0.25825 & $(1,5,1)$ & 5.03704 \\
$(1,2,1)$ & & & \\
$(1,2,2)$ & 0.87548 & $(2,2,2)$ & -0.79476 \\
$(1,2,3)$ & 0.78250 & $(2,2,3)$ & -0.65632 \\
$(1,2,4)$ & -1.99797 & $(2,2,4)$ & 1.70864 \\
$(1,2,5)$ & -0.27453 & & -0.66512 \\
$(1,2,6)$ & 2.19732 & $(2,3,2)$ & -1.94362 \\
$(1,2,7)$ & -0.51347 & $(2,3,3)$ & 3.12037 \\
$(1,3,1)$ & -1.11029 & $(2,3,4)$ & -0.19583 \\
$(1,3,2)$ & & & \\
$(1,3,3)$ & 2.57972 & $(2,4,2)$ & 6.57569 \\
$(1,3,4)$ & 1.32693 & $(3,3,3)$ & \\
$(1,3,5)$ & -3.74696 & & \\
\hline
\end{tabular}

\section{Patterns in the ordered states of the two-dimensional Heisenberg model}

\subsection{The square lattice}

For the square lattice the antiferromagnetic Heisenberg model also obeys Marshall's rule [11] in the ground state. Mermin and Wagner [3] predicted for this case that the long-range order (LRO) disappears for all temperatures $T>0$, but their results were inconclusive with respect to the existence of LRO at $T=0$. Liang c.s. [12] showed that for this case states with and without LRO exist, so one could speak of an unstable ordering, as a consequence of the existence of states with an arbitrary small distance to the exact ground state for $N \rightarrow \infty$.

This paper is primarily about Marshall states for different $r$ in the case of lattice dimension 1. For dimension 2 we restrict ourselves to $r=4$ and the total number of spins considered is $4 \times 4$ or $6 \times 6$, too small to warrant such qualitative statements as formulated by Mermin and Wagner. So we can only study in more detail the character of the special state that strictly obeys the algebraic relations following from the Marshall rule. This state in itself has very interesting properties, which in first instance are rather counter-intuitive. For a $4 \times 4$ as well as a $6 \times 6$ cell we determined the ground state for the square lattice. In both cases we used periodic boundary conditions. This ground state showed the full symmetry of the lattice but the Ising sub-states in which the 4 minority spins are arranged in a simple square with a side twice, respectively three times the elementary lattice distance, are not the dominant ones. 
Table 7

Amplitudes of the Ising configurations in the ground state with 4 minority spins for the $4 \times 4$ square

\begin{tabular}{llll}
\hline Amplitude & Total number & $\sum$ Manh. distances & Location inversions \\
\hline 0.0057675 & 24 & 8 & $(1,2,3,4) 8 /(1,2,5,6) 16$ \\
-0.0070037 & 64 & 9 & $(1,2,3,6)$ \\
0.0085573 & 192 & 10 & $(1,2,3,5) 128 /(1,2,5,8) 64$ \\
-0.013867 & 192 & 11 & $(1,2,3,8) 64 /(1,2,5,7) 128$ \\
0.014158 & 96 & 12 & $(1,2,3,10) 32 /(1,2,5,16) 64$ \\
0.015018 & 48 & 12 & $(1,2,7,8) 32 /(1,2,9,10) 16$ \\
-0.016165 & 192 & 13 & $(1,2,3,9) 64 /(1,2,5,12) 128$ \\
0.017979 & 96 & 14 & $(1,2,7,11) 64 /(1,2,9,12) 32$ \\
0.019917 & 96 & 14 & $(1,2,3,12) 32 /(1,2,5,11) 64$ \\
-0.022838 & 192 & 13 & $(1,2,7,10) 128 /(1,2,7,15) 64$ \\
0.023209 & 16 & 16 & $(1,2,11,12)$ \\
0.026888 & 16 & 12 & $(1,3,6,14)$ \\
0.027386 & 192 & 14 & $(1,2,7,9) 128 /(1,2,7,16) 64$ \\
-0.029464 & 192 & 15 & $(1,2,7,12) 128 /(1,2,9,11) 64$ \\
0.031397 & 16 & 12 & $(1,3,6,8)$ \\
0.035139 & 96 & 14 & $(1,3,6,9) 64 /(1,3,6,16) 32$ \\
0.041963 & 12 & 16 & $(1,3,9,11) 4 /(1,6,11,16) 8$ \\
-0.046583 & 64 & 15 & $(1,3,6,12)$ \\
0.054153 & 24 & 16 & $(1,3,10,12) 8 /(1,6,12,15) 16$ \\
-0
\end{tabular}

For the amplitudes of the Ising substates the sum of all Manhattan distances between the positions of the minority spins is the relevant parameter. The following "law" is suggested by our results: The larger the sum of Manhattan distances the larger the absolute value of the amplitude, with the secondary condition that the differences between the Manhattan distances for all possible pairs of minority spins should be as small as possible. On the square grid the Manhattan distance is the sum of the moduli of the differences of the $x$ - and $y$-coordinates [12].

These conditions favour a pseudo-triangular arrangement, which clearly shows by drawing the different configurations on the square grid. In Table 7 we give the numerical results for the $4 \times 4$ cell and a representant of each class of Ising configurations that have the same amplitude and the number of configurations in that class. These classes follow from the symmetry group of the systems, which contains translations, rotations and reflections. There appears for the $4 \times 4$ case, however, an additional coincidence of amplitudes that can be explained in terms of the symmetry of a four-dimensional $2 \times 2 \times 2 \times 2$ hypercube, isomorphic with the $4 \times 4$ cell. This will be explained in Section 4 .

In Table 8 for the $6 \times 6$ case we only give the amplitudes for the dominant Ising configurations, which are all related by the symmetry of the square lattice because of the fact that the extra (hypercubic) symmetry does not exist in this case. All these amplitudes should be equal and in the numerical results they only differ in the last decimal.

For the identification of the different configurations we need a numbering of the lattice points in both cells on the square lattice. This is given in Figs. 3 and 4. 
Table 8

Amplitudes of the dominant Ising configurations in the ground state with 4 minority spins for the $6 \times 6$ square

\begin{tabular}{llll}
\hline Amplitude & Configuration & Amplitude & Configuration \\
\hline 0.008805216 & $(1,10,20,29)$ & 0.008805217 & $(5,13,22,32)$ \\
0.008805218 & $(1,10,24,27)$ & 0.008805215 & $(5,15,24,32)$ \\
0.008805217 & $(1,15,24,34)$ & 0.008805216 & $(6,9,19,28)$ \\
0.008805217 & $(1,17,20,34)$ & 0.008805217 & $(6,9,23,26)$ \\
0.008805217 & $(2,11,19,28)$ & 0.008805218 & $(6,14,23,33)$ \\
0.008805217 & $(2,11,21,30)$ & 0.008805216 & $(6,16,19,33)$ \\
0.008805216 & $(2,16,19,35)$ & 0.008805217 & $(7,16,26,35)$ \\
0.008805218 & $(2,18,21,35)$ & 0.008805218 & $(7,16,30,33)$ \\
0.008805216 & $(3,12,20,29)$ & 0.008805217 & $(8,17,25,34)$ \\
0.008805218 & $(3,12,22,25)$ & 0.008805218 & $(8,17,27,36)$ \\
0.008805217 & $(3,13,22,36)$ & 0.008805215 & $(9,18,26,35)$ \\
0.008805215 & $(3,17,20,36)$ & 0.008805218 & $(9,18,28,31)$ \\
0.008805216 & $(4,7,21,30)$ & 0.008805215 & $(10,13,27,36)$ \\
0.008805217 & $(4,7,23,26)$ & 0.008805217 & $(10,13,29,32)$ \\
0.008805216 & $(4,14,23,31)$ & 0.008805217 & $(11,14,28,31)$ \\
0.008805216 & $(4,18,21,31)$ & 0.008805215 & $(11,14,30,33)$ \\
0.008805216 & $(5,8,22,25)$ & 0.008805215 & $(12,15,25,34)$ \\
0.008805216 & $(5,8,24,27)$ & 0.008805218 & $(12,15,29,32)$ \\
\hline
\end{tabular}

\section{$\begin{array}{llll}1 & 2 & 3 & 4\end{array}$ \\ $\begin{array}{llll}5 & 6 & 7 & 8\end{array}$ \\ $\begin{array}{llll}9 & 10 & 11 & 12\end{array}$}

\section{$\begin{array}{llll}13 & 14 & 15 & 16\end{array}$}

Fig. 3. Numbering of sites of the $4 \times 4$ cell of the square lattice.

$\begin{array}{cccccc}1 & 2 & 3 & 4 & 5 & 6 \\ 7 & 8 & 9 & 10 & 11 & 12 \\ 13 & 14 & 15 & 16 & 17 & 18 \\ 19 & 20 & 21 & 22 & 23 & 24 \\ 25 & 26 & 27 & 28 & 29 & 30 \\ 31 & 32 & 33 & 34 & 35 & 36\end{array}$

Fig. 4. Numbering of sites of the $6 \times 6$ cell of the square lattice. 


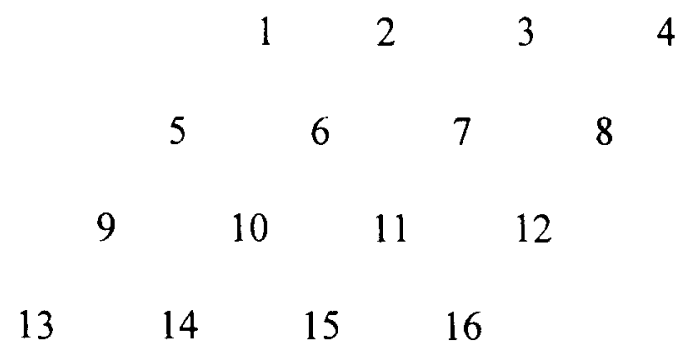

Fig. 5. Numbering of sites of the $4 \times 4$ cell of the triangular lattice.

In the last column of Table 7 we always give one representative of the one class or two classes of Ising configurations that have the same amplitude. For the case of two classes and the extra symmetry relating them we refer to Section 4 .

The "law" we formulated for the maximum value of the modulus of the amplitude may be illustrated by the 3 rows corresponding with the same sum $\sum=16$ over the Manhattan distances. For the row represented by the configuration $(1,2,11,12)$ the individual distances are: $1,4,3,3,4$ and 1 and for the ones with configurations $(1,3,9,11)$ and $(1,6,11,16)$ these distances are: $2,2,4,4,2$ and 2 and finally for $(1,3,10,12)$ and $(1,6,12,15): 2,3,3,3,3$ and 2 . The spread in the individual distances is the lowest for the last two classes. These "degenerate" classes have the same set of individual distances, which is an immediate consequence of the hypercubic symmetry.

Again the "law" for the largest amplitude is illustrated by the example of the 4 minority spins in the $6 \times 6$ square. The total sum of all Manhattan distances for all configurations of Table 8 has the maximum value 24 and all individual distances are equal. In this case there is no extra symmetry and we only give the results for these "optimal configurations".

\subsection{The triangular lattice}

This lattice shows frustration for the antiferromagnetic ordering [13-15]. This implies that Marshall's rule does not apply to the ground state and that in general the phases of the Ising components of stationary states of the antiferromagnetic Hamiltonian cannot be given. At least not on the basis of a simple algebraic recipe. Tielen [14,15], however, found a generalization of Marshall's rule, for which the set of Ising states is subdivided into three groups that have amplitudes with complex phases: $0,2 \pi / 3$ and $4 \pi / 3$. This is a kind of "Ansatz" and it can only be approximately true. The results from Tielen's method, however, are quite good in those cases in which they can be compared with the exact numbers.

In this paper we consider the ground state for the case of a minority of 4 spins down in a background of 12 spins up. We determine this ground state explicitly for a $4 \times 4$ cell with periodic boundary conditions. The cell is presented in Fig. 5 and periodic boundary conditions imply that point 1 is considered to be neighbour of $2,4,5,6,13$ and 16 . In the same way point 2 has neighbours $1,3,6,7,13$ and 14 , etc. 
Table 9

Amplitudes of the Ising configurations in the ground state with 4 minority spins for the $4 \times 4$ cell of the triangular lattice

\begin{tabular}{clll}
\hline Amplitude & Total number & $\sum$ Manhat. distances & Location inversions \\
\hline 0.00072823 & 48 & 7 & $(1,2,5,6)$ \\
-0.0016063 & 192 & 8 & $(1,2,3,6)$ \\
-0.0020138 & 32 & 9 & $(1,2,6,15)$ \\
0.0031582 & 96 & 9 & $(1,2,6,8)$ \\
0.0035462 & 48 & 11 & $(1,2,8,15)$ \\
0.0050542 & 96 & 9 & $(1,2,5,8)$ \\
-0.0052521 & 192 & 9 & $(1,2,3,5)$ \\
-0.0058062 & 96 & 10 & $(1,2,3,10)$ \\
0.0062356 & 12 & 8 & $(1,2,3,4)$ \\
-0.0076043 & 192 & 11 & $(1,2,8,10)$ \\
-0.0076988 & 96 & 10 & $(1,2,8,9)$ \\
-0.012647 & 96 & 9 & $(1,2,5,7)$ \\
0.013156 & 192 & 10 & $(1,2,5,12)$ \\
-0.014697 & 32 & 9 & $(1,2,5,16)$ \\
0.016405 & 96 & 10 & $(1,2,3,9)$ \\
0.016561 & 96 & 10 & $(1,2,5,11)$ \\
0.017079 & 48 & 10 & $(1,2,9,12)$ \\
0.035128 & 48 & 10 & $(1,2,9,10)$ \\
-0.060871 & 96 & 11 & $(1,2,9,11)$ \\
0.11115 & 12 & 12 & $(1,3,10,12)$ \\
0.26680 & 4 & 12 & $(1,3,9,11)$ \\
\hline
\end{tabular}

The Ising amplitudes for the ground state for this cell are given in Table 9. We give all the amplitudes, which again fall into classes corresponding with configurations connected by the symmetry of the lattice. In the table a class is indicated by just one representative. Again a Manhattan distance may be defined as the shortest distance along the grid between 2 lattice points, taking into consideration the connections across the boundary of the cell as a consequence of the periodic boundary conditions. Also in this case there is no "accidental" degeneracy. It is, however, rather speculative to formulate any relation between the Manhattan distances of the down spins and the (absolute) value of the amplitude, with the only exception that the dominant amplitude again corresponds with a group of Ising states with maximum Manhattan sum. This dominant amplitude corresponds with configurations that show maximum symmetry, i.e. the minority spins are on the vertices of a set of equilateral triangles.

\section{Equivalence of the $4 \times 4$ cell with the $2 \times 2 \times 2 \times 2$ hypercube}

In the discussion of the results for the $4 \times 4$ square with periodic boundary conditions we already indicated that the "accidental degeneracy" found for the amplitudes of 2 groups of Ising states can be explained in terms of the symmetry of a hypercube, i.e. the analogue of a cube in a space of dimension 4 . The similarity of these 2 systems of different dimension was already observed by other authors [16]. 
The hypercube may be "visualized" by considering Fig. 3, which shows the $4 \times 4$ square for which we have taken periodic boundary conditions. These boundary conditions imply that every point has 4 neighbours, e.g.:

1 has neighbours $2,4,5$ and 13

and 6 has neighbours $2,5,7$ and 10 .

Starting from one lattice point one can "walk" in 4 different directions, in the same way as in a four-dimensional cube. The equivalence is based on the fact that we have a small system with periodic boundary conditions: A general lattice point in the four-dimensional hypercubic lattice has 8 neighbours, instead of 4 .

Taking 4 coordinates (modulo 2) for the lattice points and let the 4 directions correspond with an increment of 1 for one of these coordinates the equivalence is immediately clear. One should take in mind, however, that the increments for steps from 1 to 2 and from 2 to 3 correspond to different four-dimensional directions.

In terms of the four-dimensional Cartesian coordinates for the points of the hypercube $\left(x_{1}, x_{2}, x_{3}, x_{4}\right)$ with $x_{i}=0$ or $1(i=1,2,3,4)$ the equivalence reads:

$$
\begin{aligned}
& 1=(0,0,0,0), 2=(1,0,0,0), 3=(1,1,0,0), 4=(0,1,0,0), \\
& 5=(0,0,1,0), 6=(1,0,1,0), 7=(1,1,1,0), 8=(0,1,1,0), \\
& 9=(0,0,1,1), 10=(1,0,1,1), 11=(1,1,1,1), 12=(0,1,1,1), \\
& 13=(0,0,0,1), 14=(1,0,0,1), 15=(1,1,0,1), 16=(0,1,0,1) .
\end{aligned}
$$

From this equivalence it should be immediately clear that e.g. the following configurations of 4 minority spins of Table 7:

$$
(1,2,3,12) \text { and }(1,2,5,11)
$$

are connected by the hypercube symmetry.

The hypercubic symmetry showing up in the $4 \times 4$ square is a mere accident without any further consequence for the understanding of ordering in two-dimensional lattices.

\section{References}

[1] H.A. Bethe, Z. Phys. 71 (1931) 205.

[2] L. Hulthén, Arkiv. Nat. Astron. Fys. A 26 (11) (1938).

[3] N.D. Mermin, H. Wagner, Phys. Rev. Lett. 17 (1966) 1133.

[4] C.J. Thompson, in: C. Domb, M.S. Green (Eds.), Phase Transitions and Critical Phenomena I, Academic Press, London, New York, 1972.

[5] F. Hund, Z. Phys, 40 (1927) 742.

[6] F. Hund, Z. Phys. 42 (1927) 93.

[7] F. Hund, Z. Phys. 52 (1929) 601.

[8] J. von Neumann, E. Wigner, Phys. Z. 30 (1929) 467.

[9] L.J.F. Broer, Physica 17 (1951) 531.

[10] W.J. Caspers, Physica 40 (1968) 125. 
[11] W. Marshall, Proc. Roy. Soc. A 232 (1955) 48.

[12] S. Liang, B. Doucot, P.W. Anderson, Phys. Rev. Lett. 61 (1988) 365.

[13] P. Fazekas, P.W. Anderson, Phil. Mag. 30 (1974) 423.

[14] W.J. Caspers, G.I. Tielen, Physica A 135 (1986) 519.

[15] G.I. Tielen, Thesis Enschede, 1994.

[16] P.N. Moustanis, A. Theophilou, S. Thanos, in: T. Lulek, W. Florek, S. Walcerz (Eds.), Symmetry and Structural Properties of Condensed Matter, Proc. 3rd SSPCM, World Scientific, Singapore, 1995. 